\title{
Proximity of the neurovascular structures during all-inside lateral meniscal repair in children: a cadaveric study
}

\author{
Yi-Meng Yen ${ }^{1 *}$ D, Peter D. Fabricant ${ }^{2}$, Connor G. Richmond ${ }^{6,7}$, Aleksei B. Dingel ${ }^{6}$, Matthew D. Milewski ${ }^{1}$, \\ Henry B. Ellis ${ }^{3}$, Philip L. Wilson ${ }^{3}$, Stephanie W. Mayer ${ }^{4}$, Theodore J. Ganley ${ }^{5}$ and Kevin G. Shea ${ }^{6}$
}

\begin{abstract}
Purpose: Meniscal repair has become increasingly common in a pediatric and adolescent population. All-inside repair techniques are utilized more often given their ease of insertion and decreased operative time required. However, there are possible risks including damage to adjacent neurovascular structures. The purpose of this study to was examine the proximity of the neurovascular structures during lateral meniscus repairs in pediatric specimens simulating a worst-case scenario.

Methods: Ten pediatric cadaveric knees (age 4-11) were utilized and simulated lateral meniscal repair through the posterior horn of the lateral meniscus and both medial and lateral to the popliteal hiatus through the body of the lateral meniscus was performed with an all-inside meniscal repair device. The distance to the popliteal artery or peroneal nerve was measured.

Results: During posterior horn repair, the average distance from the all-inside device to the popliteal artery was $1.9 \mathrm{~mm}$ $\pm 1.1 \mathrm{~mm}$. There was penetration of the artery in one specimen. During repair on the medial side of popliteal hiatus, the average distance from the all-inside device to the peroneal nerve was $3.2 \mathrm{~mm} \pm 2.0 \mathrm{~mm}$. During repair on the lateral side of popliteal hiatus, the average distance from the all-inside device to the peroneal nerve was $12.4 \mathrm{~mm} \pm 3.7 \mathrm{~mm}$.

Conclusions: This study demonstrates that the proximity of the neurovascular structures to the lateral meniscus in children is extremely close and at high risk during meniscal repair with all-inside devices. This study gives important data for the proximity of these structures during these repair techniques.
\end{abstract}

Level of evidence: Level 5 Cadaveric Study.

\section{Introduction}

In the pediatric and adolescent population, meniscal injuries are rising in incidence, in part due to increased athletic participation, expanded use of magnetic resonance imaging and improved recognition and diagnosis (Bellisari et al., 2011; Brown \& Davis, 2006; Francavilla et al., 2014). The menisci play a critical role in shock absorption, reduction of femoro-tibial contact forces and as secondary stabilizers within the knee joint. Partial or total menisectomies can lead to early joint degeneration and resultant osteoarthritis (Lanzer \& Komenda, 1990;

\footnotetext{
* Correspondence: Yi-Meng.Yen@childrens.harvard.edu

'Boston Children's Hospital, Division of Sports Medicine, Department of

Orthopaedics, Harvard Medical School, Boston, MA, USA

Full list of author information is available at the end of the article
}

McDermott \& Amis, 2006; Shoemaker \& Markolf, 1986). The trend has therefore been towards repair of meniscal tears, particularly in the pediatric and adolescent athlete.

The inside-out meniscal repair remains the gold standard for meniscal fixation, but can be associated with an increased surgical time and the need for an additional posterolateral or posteromedial incision (Lembach \& Johnson, 2014; Woodmass et al., 2017). All-inside fixation has been advanced in the arthroscopic field due to the ease of implantation and decreased surgical time. The newest generation of these devices is designed to incorporate strength of the inside-out repair with the advantages of the all-inside technique (Fillingham et al., 2017). However, there is still risk of neurovascular injury 
during the surgical management of lateral meniscal tears. While there have been some anatomic studies on this in the adult literature (Abouheif et al., 2011; Cohen et al., 2007; Cuellar et al., 2015; Deutsch et al., 1999), to our knowledge, there are no comparable studies in younger patients. The purpose of this study was to evaluate the proximity of the neurovascular structures when using an all-inside meniscal repair device in pediatric cadaveric specimens. This was performed in a way to give the worst-case scenario during a simulated repair. Our hypothesis was that in children, the neurovascular structures are extremely close to the lateral meniscus.

\section{Methods}

The study was performed on ten fresh-frozen cadaveric specimens that included the full knee joint from the distal half of the femur to the proximal half of the tibia including all surrounding tissues. There were 6 male cadavers and 4 female cadavers ranging from four to eleven years in age. The knees were fresh frozen and thawed which allowed for free motion at the joint.

\section{Specimen preparation}

All cadaveric specimens were dissected in a similar manner. The tibial plateau width and lngeth were also recorded. The knee extensor mechanism was turned downwards by freeing the quadriceps tendon and quadriceps along with and arthrotomies performed on the medial and lateral sides of the patellar tendon. The MCL and LCL were partially released to allow full visualization of the lateral meniscus. The all-inside device used for this study was a curved FasT-Fix $360^{\circ}$ (Smith-Nephew, Andover, MA) without a depth limiter to allow for full penetration of the device (Fig. 1).

\section{Posterior horn of the lateral meniscus}

The all inside device was placed into the joint through a simulated anterolateral portal and inserted at the visible edge (within 1-2 $\mathrm{mm}$ ) of the posterior root of the lateral meniscus. In order to simulate a lateral meniscus tear near the posterior root, the curved tip was aimed medially and, thus, towards the neurovascular bundle. Following implantation, the tip of the needle was left in situ and then was identified through a direct posterior approach with the knee in flexion. The nearest measurement of the needle to the popliteal artery was taken.

Medial side of popliteal hiatus. The all-inside device was again placed into the joint through a simulated anterolateral portal and inserted at the most medial corner of the popliteal hiatus. The curved tip was aimed laterally towards the peroneal nerve. A posterolateral dissection was performed to identify the needle and tip. The nearest measurement of the needle to the common peroneal nerve was recorded.

\section{Lateral side of popliteal hiatus}

The all inside device was placed into the joint through a simulated anteromedial arthroscopy portal and inserted at the lateral corner of the popliteal hiatus. The curved tip of the needle was aimed medially towards the common peroneal nerve. Measurement of the needle to the peroneal nerve was taken.

\section{Measurements}

All the measurements were done by a single fellowshiptrained surgeon. A digital caliper with a nominal precision of $0.01 \mathrm{~mm}$ was used in all measurements from the position of the needle to the neurovascular structures. Three caliper measurements were performed on each specimen at each of the three locations of the all inside

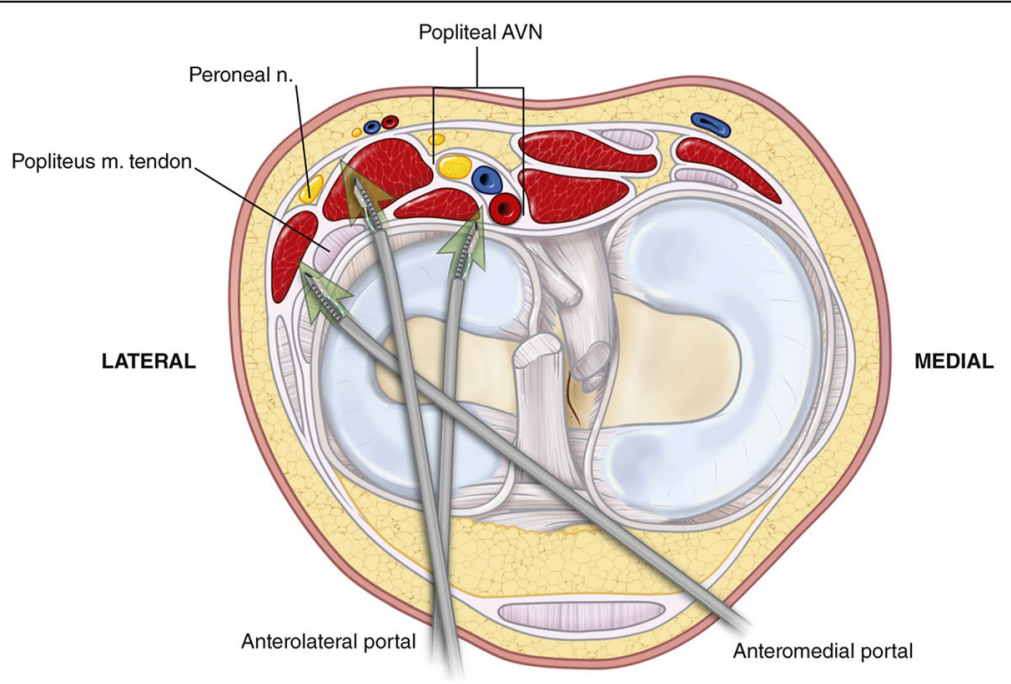

Fig. 1 Axial schematic of the all-inside meniscal repair devices and the neurovascular structures of a left knee 
device needle and the average was used as the final measure. The knee was held at 90 degrees of flexion in all cases to simulate arthroscopic positioning in the figure-of-four position.

\section{Results}

The dimensions of the each specimen and measurements between the neurovascular structures and the all inside meniscal devices are listed in Table 1.

\section{Distance from posterior root to popliteal artery}

The average distance from the all-inside device to the popliteal artery was $1.9 \mathrm{~mm} \pm 1.1 \mathrm{~mm}$ (SD). There was penetration of the artery in a specimen of a 4 year-old knee specimen (Fig. 2). The farthest distance to the artery was $3.8 \mathrm{~mm}$ in a 10 year-old knee.

\section{Medial side of popliteal Hiatus to the common peroneal nerve}

The average distance from the all-inside device to the peroneal nerve was $3.2 \mathrm{~mm} \pm 2.0 \mathrm{~mm}$ (SD) (Fig. 3). The closest distance to the nerve was $0.4 \mathrm{~mm}$ in a 4 year-old knee specimen while the farthest distance to the nerve was $6.6 \mathrm{~mm}$ in a 10 year-old knee.

\section{Lateral side of popliteal Hiatus to the common peroneal nerve}

The average distance from the all-inside device to the peroneal nerve was $12.4 \mathrm{~mm} \pm 3.7 \mathrm{~mm}$ (SD). The closest distance to the nerve was $5.5 \mathrm{~mm}$ in a 4 year-old knee. The farthest distance to the nerve was $17.6 \mathrm{~mm}$ in a 9 year-old knee.

Table 1 Age of specimens, Tibial plateau dimensions, and distance from meniscal repair device to neurovascular structures. (All measurements presented in $\mathrm{mm}$ )

\begin{tabular}{llllll}
\hline $\begin{array}{l}\text { Age/ } \\
\text { Sex }\end{array}$ & $\begin{array}{l}\text { Tibial } \\
\text { Plateau } \\
\text { Length }\end{array}$ & $\begin{array}{l}\text { Tibial } \\
\text { Plateau } \\
\text { Width }\end{array}$ & $\begin{array}{l}\text { Distance } \\
\text { to } \\
\text { Popliteal } \\
\text { Artery }\end{array}$ & $\begin{array}{l}\text { Distance to } \\
\text { Peroneal Nerve } \\
\text { (Medial Popliteus) }\end{array}$ & $\begin{array}{l}\text { Distance to } \\
\text { Peroneal } \\
\text { Nerve (Lateral } \\
\text { Popliteus) }\end{array}$ \\
\hline 4M & 30.8 & 20.2 & 0.0 & 1.0 & 5.5 \\
4F & 30.7 & 20.4 & 1.8 & 0.4 & 15.1 \\
7F & 30.4 & 20.3 & 1.7 & 4.4 & 8.2 \\
9M & 60.2 & 30.6 & 2.9 & 5.0 & 17.6 \\
9F & 50.1 & 30.2 & 1.3 & 3.7 & 11.6 \\
9F & 50.1 & 20.9 & 2.9 & 1.2 & 13.1 \\
9M & 60.1 & 30.4 & 0.9 & 1.9 & 10.6 \\
10 M Unable & Unable & 3.8 & 4.4 & 16.3 \\
& to collect & to collect & & & 13.4 \\
10 M & 60.4 & 30.8 & 1.4 & 6.6 & 12.7 \\
$11 \mathrm{M}$ & 60.7 & 30.9 & 2.1 & 3.0 & \\
\hline
\end{tabular}

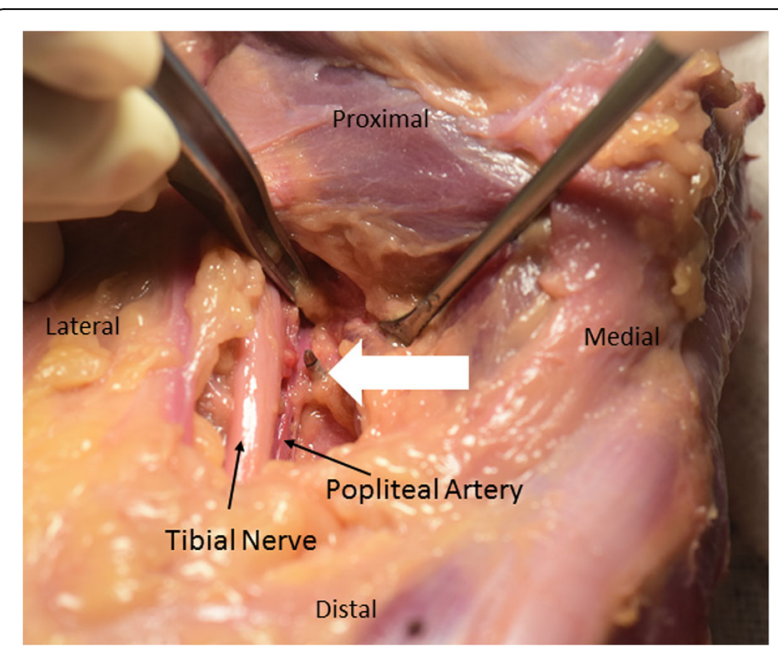

Fig. 2 Viewing from posterior to anterior of a left knee, showing the needle tip (white arrow) just adjacent to the popliteal artery and the proximity to the tibial nerve

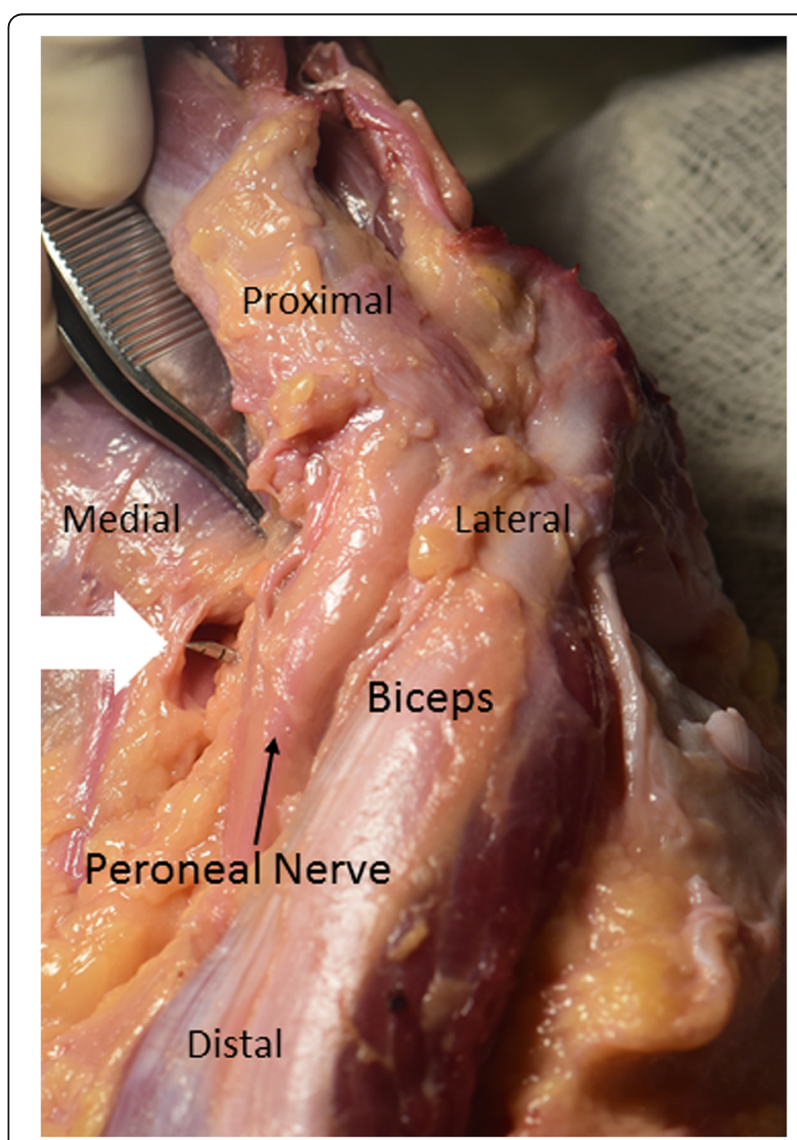

Fig. 3 Viewing from posterior to anterior of a right knee, showing the needle tip (white arrow) adjacent to the peroneal nerve while performing a repair on the medial side of the popliteal hiatus 


\section{Discussion}

The incidence of neurovascular injury during all-inside meniscal repair is reportedly very low (Bernard et al., 1994). In a study of 375,000 knee arthroscopies, there were 9 injuries to the popliteal artery (Complications in arthroscopy, 1986). In a similar study of 118,590 arthroscopic procedures, there were 6 cases of injury to the popliteal artery. (Complications of arthroscopy and arthroscopic surgery, 1985) There have been some case reports of common peroneal nerve injury after lateral meniscal repair (Anderson \& LaPrade, 2009; Krivic et al., 2003). However, to the author's knowledge, the incidence of neurovascular complications following meniscal repair in children has not been reported. While, the overall number of procedures in children per year is lower than in adults, meniscal repair in children may be more common. As the overall dimensions of a pediatric knee are smaller, the proximity of the neurovascular structures may place them at greater risk during meniscal repair.

This study was conducted in order to determine the proximity of the neurovascular structure in a "worstcase" scenario utilizing an all-inside meniscal repair device intentionally aimed towards the neurovascular structures. If the all-inside device were utilized in as recommended, this would bring the device further from the neurovascular structures and therefore safer. The popliteal artery is located near the posterior horn of the lateral meniscus and in extension is only separated by the posterior capsule by a small layer of fat. The common peroneal nerve is located posterior to the biceps femoris tendon and is located next to the popliteal hiatus on the medial side. In adult studies, when the distance between the repair device and the neurovascular structures was $10 \mathrm{~mm}$, this was considered at risk, when the distance was less than $5 \mathrm{~mm}$, this risk was considered high (Abouheif et al., 2011; Cuellar et al., 2015; Deutsch et al., 1999). With this definition, lateral meniscal repair of the posterior horn near the root in children should be considered especially high risk as the popliteal artery is within $5 \mathrm{~mm}$ of the suturing device. Direct penetration of the artery was seen in one specimen of a 4 year-old child. The common peroneal nerve is also at high risk when repairing the meniscus at the medial edge of the popliteal hiatus. The device was essentially adjacent to the peroneal nerve in a specimen of a 4 year-old child.

There have been several studies performed in adult cadaveric specimens that examined the safety of lateral meniscal repair. Cohen et al. examined the use of 2 repair devices in the posterior horn of the lateral meniscus and found that the tip of an all-inside device without the penetration limiter was within $3 \mathrm{~mm}$ of the artery (Cohen et al., 2007). Abouheif et al. found damage to the popliteal vessels and peroneal nerve in 33\% and 10\% of their 31 knees (Abouheif et al., 2011). Cuellar et al. reported that the risk of lateral meniscal repair is safer at $90^{\circ}$ and worse with extension of the knee (Cuellar et al., 2015). Recently, an MRI based study demonstrated the significant risk to the neurovascular structures with repair of the lateral meniscus (Gupta et al., 2018).

This study has several limitations inherent to cadaveric studies. While the insertion of the device was done at $90^{\circ}$ to simulate the figure-of-four position during arthroscopic surgery, this was performed in a dry environment without fluid to distend the joint. The study was performed with a curved all-inside device with an angle of $29^{\circ}$ intentionally aimed towards the neurovascular bundle. We wished to perform a "worst-case" scenario, however, if the direction of the curve of the device was altered or if a straight device was utilized, this would likely decrease the risk to the neurovascular structures. Additionally, we simulated the anterolateral and anteromedial portals by positioning the devices adjacent to the patellar tendon. Variation in true placement of these portals can alter the trajectory of the device. This study utilized the suture device without the use of the limiter which does not take into account the anterior-posterior position of the neurovascular structures. The proximity of the neurovascular structures in the antero-posterior direction is age-dependent and is as close as $4 \mathrm{~mm}$ (submitted) It is likely that positioning the penetration limiter less than $20 \mathrm{~mm}$ may decrease the risk of penetrating the peroneal nerve or popliteal artery, but this was not addressed in this study. The dissection posteriorly and posteolaterally were performed after device insertion and may have allowed for movement of the tissues. Although, the fresh freezing and thaw method allows for near normal elasticity of the tissue, dissection of the tissue may inevitably alter the true relationship of the meniscus with the neurovascular structures.

This study demonstrates that the proximity of the neurovascular structures to all-inside devices inserted in the lateral meniscus in children are closer than has been previously demonstrated in some adult studies. As the incidence of meniscal repair is rising in children, great care must be taken when performing lateral meniscal repair using all-inside devices. Future research should simulate actual arthroscopic conditions and the proper clinical use of all-inside meniscal repair devices in pediatric specimens.

\section{Acknowledgments}

Todd Huft, Lisa Houck, and Tom Cycotoa, CEO, of Allosource, Centennial, Colorado. Their generous donation of these specimens and use of their facilities allowed for this study. Smith and Nephew Endoscopy donated the all-inside devices that were used in this cadaveric study. We would like to honor, and acknowledge the families who made the gifts of donation to our study group. Their donations have made this research possible, and will contribute to lives of other families and their children.

\section{Authors' contributions}

Each author listed above had either substantial contributions to the conception or design of the work; or the acquisition, analysis, or 
interpretation of data for the work; AND drafting the work or revising it critically for important intellectual content; AND approved the final version of the manuscript; AND are in agreement to be accountable for all aspects of the work in ensuring that questions related to the accuracy or integrity of any part of the work are appropriately investigated and resolved.

\section{Competing interests}

The authors declare that they have no competing interests.

\section{Publisher's Note}

Springer Nature remains neutral with regard to jurisdictional claims in published maps and institutional affiliations.

\section{Author details}

'Boston Children's Hospital, Division of Sports Medicine, Department of Orthopaedics, Harvard Medical School, Boston, MA, USA. ${ }^{2}$ Hospital for Special Surgery, New York, NY, USA. ${ }^{3}$ Texas Scottish Rite Hospital, University of Texas Southwestern, Dallas, TX, USA. ${ }^{4}$ Children's Hospital Colroado, Aurora, CO, USA. ${ }^{5}$ Children's Hospital Philadelphia, University of Pennsylvania School of Medicine, Philadelphia, PA, USA. ${ }^{6}$ Department of Orthopedic Surgery, Stanford University, Stanford, CA, USA. University of New England, College of Osteopathic Medicine, Biddeford, ME, USA.

Received: 18 August 2018 Accepted: 27 November 2018

Published online: 18 December 2018

\section{References}

Abouheif MM, Shibuya H, Niimoto T, Kongcharoensombat W, Deie M, Adachi N et al (2011) Determination of the safe penetration depth during all-inside meniscal repair of the posterior part of the lateral meniscus using the FasTfix suture repair system. Knee Surg Sports Traumatol Arthrosc 19:1868-1875

Anderson AW, LaPrade RF (2009) Common peroneal nerve neuropraxia after arthroscopic inside-out lateral meniscus repair. J Knee Surg 22:27-29

Bellisari G, Samora W, Klingele K (2011) Meniscus tears in children. Sports Med Arthrosc Rev 19:50-55

Bernard M, Grothues-Spork M, Georgoulis A, Hertel P (1994) Neural and vascular complications of arthroscopic meniscal surgery. Knee Surg Sports Traumato Arthrosc 2:14-18

Brown T, Davis J (2006) Meniscal injury in the skeletally immature patient. In: Micheli L, Kocher M (eds) The pediatric and adolescent knee. SaundersElsevier

Cohen SB, Boyd L, Miller MD (2007) Vascular risk associated with meniscal repair using Rapidloc versus FasT-fix: comparison of two all-inside meniscal devices. J Knee Surg 20:235-240

Complications in arthroscopy (1986): The knee and other joints. Committee on Complications of the Arthroscopy Association of North America. Arthroscopy 2:253-258

Complications of arthroscopy and arthroscopic surgery (1985): results of a national survey. Committee on Complications of Arthroscopy Association of North America. Arthroscopy 1:214-220

Cuellar A, Cuellar R, Cuellar A, Garcia-Alonso I, Ruiz-lban MA (2015) The effect of knee flexion angle on the neurovascular safety of all-inside lateral meniscus repair: a cadaveric study. Arthroscopy 31:2138-2144

Deutsch A, Wyzykowski RJ, Victoroff BN (1999) Evaluation of the anatomy of the common peroneal nerve. Defining nerve-at-risk in arthroscopically assisted lateral meniscus repair. Am J Sports Med 27:10-15

Fillingham YA, Riboh JC, Erickson BJ, Bach BR Jr, Yanke AB (2017) Inside-out versus all-inside repair of isolated meniscal tears: an updated systematic review. Am J Sports Med 45:234-242

Francavilla ML, Restrepo R, Zamora KW, Sarode V, Swirsky SM, Mintz D (2014) Meniscal pathology in children: differences and similarities with the adult meniscus. Pediatr Radiol 44:910-925 quiz 907-919

Gupta H, Ghasi RG, Kataria H, Jain V, Shankar V, Daripa RK et al (2018) Popliteal neurovascular bundle is safe during inside-out repair of medial meniscus without a safety incision. In: Knee Surg sports Traumatol Arthrosc

Krivic A, Stanec S, Zic R, Budi S, Milanovic R, Stanec Z (2003) Lesion of the common peroneal nerve during arthroscopy. Arthroscopy 19:1015-1018

Lanzer WL, Komenda G (1990) Changes in articular cartilage after meniscectomy. Clin Orthop Relat Res 252;41-48
Lembach M, Johnson DL (2014) Meniscal repair techniques required for the surgeon performing anterior cruciate ligament reconstruction. Orthopedics 37:617-621

McDermott ID, Amis AA (2006) The consequences of meniscectomy. J Bone Joint Surg Br 88:1549-1556

Shoemaker SC, Markolf KL (1986) The role of the meniscus in the anteriorposterior stability of the loaded anterior cruciate-deficient knee. Effects of partial versus total excision. J Bone Joint Surg Am 68:71-79

Woodmass JM, LaPrade RF, Sgaglione NA, Nakamura N, Krych AJ (2017) Meniscal repair: reconsidering indications, techniques, and biologic augmentation. J Bone Joint Surg Am 99:1222-1231

\section{Submit your manuscript to a SpringerOpen ${ }^{\circ}$ journal and benefit from:}

- Convenient online submission

- Rigorous peer review

- Open access: articles freely available online

- High visibility within the field

- Retaining the copyright to your article

Submit your next manuscript at $\boldsymbol{\nabla}$ springeropen.com 